\title{
Membrane and capillary component of the lung Diffusion capacity in obesity and bariatric Surgery
}

\author{
NA Zhou, Corentin Scoubeau, Vitalie Faoro
}

European Respiratory Journal 2021 58: PA3215; DOI: 10.1183/13993003.congress2021.PA3215

Introduction: A linear and inverse association between the degree of obesity and the respiratory function has previously been attributed to altered respiratory system compliance and mechanics. The purpose of this study is to evaluate the impact of obesity and weight loss on the membrane and the capillary component of lung diffusion capacity.

Methods: Measurements of lung diffusing capacity (DL) for carbon monoxide (CO) and nitric oxide (NO) during a single breath in 13 obese subjects before and 6 months after bariatric surgery (Body Mass Index (BMI): $40 \pm 4$ vs $31 \pm 3 \mathrm{~kg} / \mathrm{m} 2$ ) were compared to age-, sex-, height-, and ethnic-matched healthy control subjects. Body composition was determined by dual-energy $\mathrm{X}$-ray absorptiometry.

Results: Obesity was associated with decreased alveolar volume (VA), DLNO and membrane diffusion capacity $(\mathrm{Dm})$ but with unaffected capillary volume $(\mathrm{Vc})$, rate of uptake of $\mathrm{NO}(\mathrm{KNO})$ and $\mathrm{CO}$ (KCO). Bariatric surgery and $32 \%$ fat mass loss increased VA, DLNO and Dm. Visceral adipose tissue loss was correlated with post-surgery increase in DLNO/DLCO and Dm/Nc ratios $(r=-0.55, p<0.05)$.

$$
\text { Controls Obesity Pre-surgery Post-surgery }
$$

$\begin{array}{lccc}\text { DLNO (ml.min-1.mmHg-1) } & 139 \pm 19 & 124 \pm 29 & 138 \pm 36^{*} \\ \text { DLCOcor (ml.min-1.mmHg-1) } & 27.3 \pm 3.5 & 22.7 \pm 4.1^{\circ \circ} & 24.7 \pm 5.8 \\ \text { VA (l) } & 6.3 \pm 0.9 & 5.5 \pm 1.2 & 6.0 \pm 1.2^{*} \\ \text { Vc (ml) } & 75.1 \pm 13.1 & 62 \pm 10^{\circ \circ} & 66 \pm 15 \\ & & & 196 \pm 99^{*} \\ \text { Dm (ml.min-1.mmHg-1) } & 173 \pm 55 & 146 \pm 46 & \end{array}$

${ }^{*} p<0.05$ : pre- vs post-surgery, ${ }^{\circ 0} p<0.01$ : vs controls

Conclusion: Obesity affects lung diffusion capacity by a reduced VA mainly affecting the membrane diffusion capacity, probably related to excessive thoracic and abdominal adipose tissue deposit. The observed deficits were restored 6 months after surgery induced fat mass loss. 